\title{
Effect of Material Nonlinearity on Large Deflection of Variable-Arc-Length Beams Subjected to Uniform Self-Weight
}

\author{
Chainarong Athisakul, ${ }^{1}$ Boonchai Phungpaingam, ${ }^{2}$ \\ Gissanachai Juntarakong, ${ }^{1}$ and Somchai Chucheepsakul ${ }^{1}$ \\ ${ }^{1}$ Department of Civil Engineering, Faculty of Engineering, \\ King Mongkut's University of Technology Thonburi, Bangkok 10140, Thailand \\ ${ }^{2}$ Department of Civil Engineering, Rajamangala University of Technology Thanyaburi, \\ Pathum-thani 12110, Thailand \\ Correspondence should be addressed to Chainarong Athisakul, chainarong.ath@kmutt.ac.th \\ and Somchai Chucheepsakul, somchai.chu@kmutt.ac.th
}

Received 21 June 2011; Accepted 22 October 2011

Academic Editor: Paulo Batista Gonçalves

Copyright (C) 2012 Chainarong Athisakul et al. This is an open access article distributed under the Creative Commons Attribution License, which permits unrestricted use, distribution, and reproduction in any medium, provided the original work is properly cited.

This paper presents a large deflection of variable-arc-length beams, which are made from nonlinear elastic materials, subjected to its uniform self-weight. The stress-strain relation of materials obeys the Ludwick constitutive law. The governing equations of this problem, which are the nonlinear differential equations, are derived by considering the equilibrium of a differential beam element and geometric relations of a beam segment. The model formulation presented herein can be applied to several types of nonlinear elastica problems. With presence of geometric and material nonlinearities, the system of nonlinear differential equations becomes complicated. Consequently, the numerical method plays an important role in finding solutions of the presented problem. In this study, the shooting optimization technique is employed to compute the numerical solutions. From the results, it is found that there is a critical self-weight of the beam for each value of a material constant $n$. Two possible equilibrium configurations (i.e., stable and unstable configurations) can be found when the uniform self-weight is less than its critical value. The relationship between the material constant $n$ and the critical self-weight of the beam is also presented.

\section{Introduction}

The variable-arc-length (VAL) beam is an elastica problem, which can be applied to a variety of applications such as marine risers / pipes, marine cables, and also paper feeders. According to a fast growing in these industries, several new materials are developed for the sake of safety and economy. Most of these materials possess a nonlinear stress-strain relation. Consequently, the study in effects of material nonlinearity becomes important and interesting 
research topics. In the literature, the material law of VAL beams is limited to the linear relationship between stress and strain [1-8], where it might not be suitable for the other classes of materials. In practices, the stress-strain curve for any elastic material exhibits the linear relationship within a narrow range. Afterwards, the nonlinear relationship can be observed. Hence, in the large deflection analysis, the material nonlinearity should be taken into account for more practical results. However, the publications concerning this kind of problem are very limited. Moreover, most of publications in this area give their attention to only the problem of large deflection of cantilever beams [9-12]. Therefore, it is very interesting to explore the effects of material nonlinearity on the large deflection behavior of the proposed VAL beam.

From the authors' experience, the VAL beam obeying the Hooke's law subjected to the uniform self-weight can lose its stability at a critical load $[6,8]$. Thus, it is interesting to investigate the behaviors of the VAL beam (i.e., equilibrium shapes and their stability) made from the Ludwick's material under the uniform self-weight. There is a shortcoming to the case of straight columns made from the Ludwick's material where the critical load becomes zero or infinity when the nonlinearity in terms of strain is appeared [13]. This is because, at an infinitesimal deflection, the slope of the stress-strain curve of the Ludwick's material becomes zero or infinity when the degree of material nonlinearity is presented. However, that is not the case for this study since the critical load of the VAL beam under the influence of the uniform selfweight is expected to take place at a finite value of the deflection.

In this paper, the VAL beam made from the Ludwick's material subjected to the uniform self-weight is investigated. One end of the beam is attached to the hinged support, while the other end is placed on the frictionless roller support. The length of the VAL beam is allowed to increase by sliding on the roller support. The governing differential equations of the problem are established from the equilibrium of the beam segment, the constitutive equation, and the geometric relations. The shooting method is employed for evaluating the numerical results of the problem. From the results, the load-deflection curves are plotted for studying the behaviors of the VAL beam. The stability of the VAL beam is interpreted from the loaddeflection diagrams (i.e., the slope of equilibrium paths). The positive, zero, and negative slopes are related to stable, critical, and unstable states, respectively. Furthermore, the critical self-weight depending on the degree of material nonlinearity is also highlighted herein.

\section{Problem Description}

The variable arc-length beam of span length $L$ is supported by a pin joint at end $A$ and by a frictionless roller at end $B$ as shown in Figure 1. According to the uniform self-weight per unit arc-length of the beam $w$, the beam can experience a large deflection by sliding over the frictionless support $B$. Therefore, a total arc-length $s_{t}$ is an unknown variable to be determined. The beam is made from a nonlinearly elastic material, where the stress-strain relation is represented by the Ludwick constitutive law [13] as follows:

$$
\sigma(\varepsilon)= \begin{cases}E|\varepsilon|^{1 / n} ; & \varepsilon \geq 0 \\ -E|\varepsilon|^{1 / n} ; & \varepsilon<0\end{cases}
$$

where $\sigma$ and $\varepsilon$ stand for the stress and strain, respectively. The constants $E$ and $n, n>0$, are material properties, where the material nonlinearity can be observed when $n \neq 1$. This stressstrain relation has been used primarily for work hardening metals [11, 14]. 


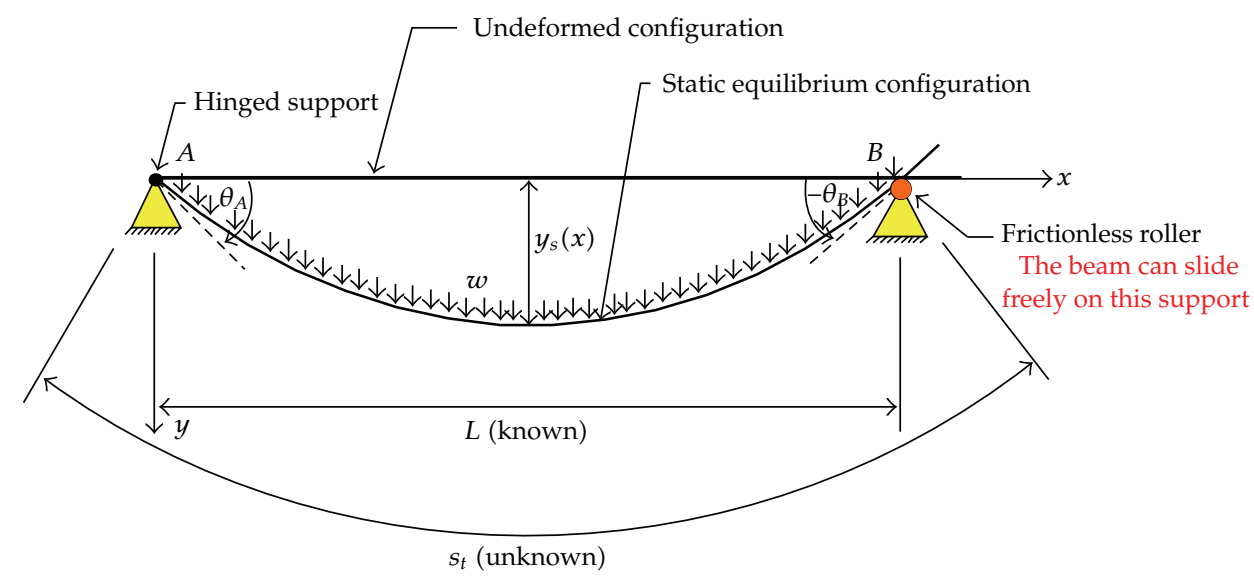

Figure 1: The variable-arc-length beam subjected to the uniform self-weight.

\section{Model Formulation}

The model formulation used in this study is developed based on the following assumptions.

(a) An axial movement is unrestrained at the frictionless roller; therefore, the effect of the axial deformation is not included in this analysis.

(b) The beam material is assumed to be homogeneous and isotropic.

(c) The stress-strain relation of the beam material obeys the Ludwick constitutive law.

(d) A cross-section of the beam still remains plane and perpendicular to the neutral axis after deformations.

(e) The shear deformation is small in comparison with the axial deformation and can be neglected.

\subsection{Moment-Curvature Relation}

According to the nonlinear stress-strain relation, the bending moment can be expressed in terms of the normal stress as follows:

$$
M=\int \sigma y d A
$$

The normal strain-curvature relation is

$$
\varepsilon=-\frac{y}{\rho}=-\kappa y .
$$

Substituting (2.1) and (3.2) into (3.1), one obtains the moment-curvature relation as follows:

$$
M(s)=-\int E \kappa^{1 / n} y^{(n+1) / n} d A=-E I_{n} \mathcal{\kappa}^{1 / n}=-E I_{n}\left(\frac{d \theta}{d s}\right)^{1 / n},
$$




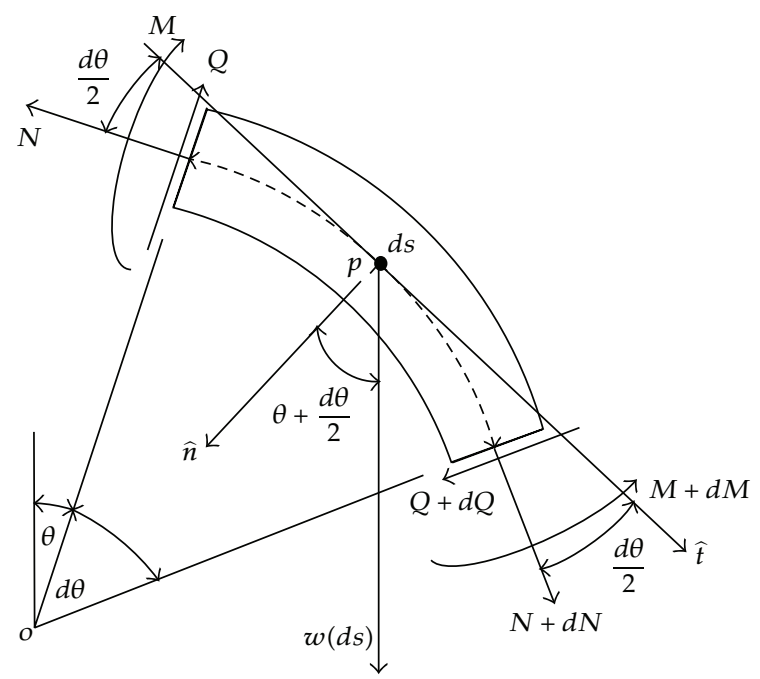

Figure 2: Free-body diagram of the differential beam element.

where $I_{n}$ represents a cross-sectional property of a beam made from the Ludwick type of material, $\kappa=d \theta / d s$ represents the curvature, and $\theta$ is an angle measured from the horizontal axis to the neutral axis of the beam. For a special case of a rectangular beam of width $b$ and depth $h$, the cross-sectional property $I_{n}$ can be expressed as follows [13]:

$$
I_{n}=\int y^{(n+1) / n} d A=\left(\frac{1}{2}\right)^{(n+1) / n}\left(\frac{n}{2 n+1}\right) b h^{((2 n+1) / n)} .
$$

One can find that if a linear elastic material $(n=1)$ is used, $I_{n}$ is equal to the moment of inertia of the rectangular beam $\left(I_{n=1}=b h^{3} / 12\right)$.

\subsection{Governing Equations and Boundary Conditions}

Considering a system of forces acting on a beam segment (Figure 2), the internal forces in $\widehat{n}$, and $\hat{t}$ directions are in equilibrium. The equilibrium of moments is taken about the point $P$, one obtains the internal forces and moment equations as follows:

$$
\frac{d M}{d s}=Q, \quad \frac{d Q}{d s}=-N \frac{d \theta}{d s}-w \cos \theta, \quad \frac{d N}{d s}=Q \frac{d \theta}{d s}-w \sin \theta
$$

where $Q$ and $N$ denote the shear force and the axial force, respectively. The geometric relations between the Cartesian coordinates $(x, y)$ and the intrinsic coordinates $(s, \theta)$ are

$$
\frac{d x}{d s}=\cos \theta, \quad \frac{d y}{d s}=\sin \theta
$$


The nonlinear governing differential equations of this problem are described by (3.3), (3.5), and (3.6). For the sake of generality and numerical calculation, the following nondimensional quantities are introduced.

$$
\begin{gathered}
s^{*}=\frac{s}{s_{t}}, \quad \widehat{x}=\frac{x}{L}, \quad \widehat{y}=\frac{y}{L}, \quad \widehat{s}=\frac{s}{L}, \\
\widehat{M}=\frac{M L^{1 / n}}{E I_{n}}, \quad \widehat{Q}=\frac{Q L^{(n+1) / n}}{E I_{n}}, \quad \widehat{N}=\frac{N L^{(n+1) / n}}{E I_{n}}, \quad \widehat{w}=\frac{w L^{(2 n+1) / n}}{E I_{n}} .
\end{gathered}
$$

By utilizing (3.7), the governing equations ((3.3), (3.5), and (3.6)) can be rearranged in the following nondimensional forms:

$$
\begin{gathered}
\frac{d \theta}{d s^{*}}=-\widehat{s}_{t} \widehat{M}^{n}, \quad \frac{d \widehat{M}}{d s^{*}}=\widehat{s}_{t} \widehat{Q}, \quad \frac{d \widehat{Q}}{d s^{*}}=-\widehat{N} \frac{d \theta}{d s^{*}}-\widehat{s}_{t} \widehat{w} \cos \theta, \quad \frac{d \widehat{N}}{d s^{*}}=\widehat{Q} \frac{d \theta}{d s^{*}}-\widehat{s}_{t} \widehat{w} \sin \theta, \\
\frac{d \widehat{x}}{d s^{*}}=\widehat{s}_{t} \cos \theta, \quad \frac{d \widehat{y}}{d s^{*}}=\widehat{s}_{t} \sin \theta .
\end{gathered}
$$

The boundary conditions of the variable-arc-length beam at the support $A$ are

$$
s^{*}=0, \quad \widehat{x}=0, \quad \widehat{y}=0, \quad \widehat{M}=0, \quad \theta=\theta_{A}, \quad \widehat{Q}=\widehat{Q}_{A}, \quad \widehat{N}=\widehat{N}_{A},
$$

and those at the support $B$ are

$$
s^{*}=1, \quad \widehat{x}=1, \quad \widehat{y}=0, \quad \widehat{M}=0, \quad \theta=\theta_{B}, \quad \widehat{Q}=\widehat{Q}_{B}, \quad \widehat{N}=0 .
$$

\section{Solution Method}

In this paper, the shooting method is employed to obtain the numerical solutions. The numerical procedure can be summarized in the following steps.

Step 1. Set the initial step size of integration and the initial values of $\widehat{x}\left(s^{*}\right), \widehat{y}\left(s^{*}\right), \theta\left(s^{*}\right), \widehat{M}\left(s^{*}\right)$, $\widehat{Q}\left(s^{*}\right)$, and $\widehat{N}\left(s^{*}\right)$ at $s^{*}=1$.

Step 2. Assign $\theta(1)=\theta_{B}$ and estimate the values of $s_{t}^{*}, \widehat{Q}_{B}$, and $\widehat{w}$ for the first iteration from the small displacement theory.

Step 3. Integrate (3.8) from $s^{*}=1$ to 0 by using the fourth-order Runge-Kutta method.

Step 4. Minimize the error norm according to end conditions at support $A$, with respect to $s_{t}^{*}$, $\widehat{Q}_{B}$, and $\widehat{w}$. Therefore, the objective function $\Phi$ for the minimization process is

$$
\underset{\widehat{s}_{t}, \widehat{Q}_{B}, \widehat{w}}{\operatorname{Min}} \Phi=|\widehat{x}(0)|+|\widehat{y}(0)|+|\widehat{M}(0)| .
$$

The Newton-Raphson iterative scheme is utilized to minimize the objective function $\Phi$. 
Table 1: Numerical values of $\theta_{A}, \widehat{w}, \widehat{y}_{\max }, \widehat{s}_{t}$, and $\widehat{M}_{\max }$ for $n=1,2,3$, and 4 .

\begin{tabular}{|c|c|c|c|c|c|}
\hline$n$ & $\theta_{A}($ radian $)$ & $\widehat{w}$ & $\widehat{y}_{\max }$ & $\widehat{s}_{t}$ & $\widehat{M}_{\max }$ \\
\hline \multirow{7}{*}{1} & 0.00 & 0.0000 & 0.0000 & 1.0000 & 0.0000 \\
\hline & 0.25 & 5.4504 & 0.0790 & 1.0154 & 0.7432 \\
\hline & 0.50 & 8.1451 & 0.1632 & 1.0645 & 1.4417 \\
\hline & 0.75 & 7.4046 & 0.2589 & 1.1565 & 2.0358 \\
\hline & 1.00 & 4.6549 & 0.3751 & 1.3108 & 2.4518 \\
\hline & 1.25 & 1.8750 & 0.5277 & 1.5658 & 2.6186 \\
\hline & 1.50 & 0.2313 & 0.7499 & 2.0062 & 2.4891 \\
\hline \multirow{7}{*}{2} & 0.00 & 0.0000 & 0.0000 & 1.0000 & 0.0000 \\
\hline & 0.25 & 7.0095 & 0.0872 & 1.0181 & 0.9656 \\
\hline & 0.50 & 7.3203 & 0.1820 & 1.0766 & 1.3503 \\
\hline & 0.75 & 5.3230 & 0.2940 & 1.1905 & 1.6084 \\
\hline & 1.00 & 2.8044 & 0.4384 & 1.3917 & 1.7576 \\
\hline & 1.25 & 0.9533 & 0.6468 & 1.7535 & 1.7859 \\
\hline & 1.50 & 0.0948 & 1.0026 & 2.4752 & 1.6714 \\
\hline \multirow{7}{*}{3} & 0.00 & 0.0000 & 0.0000 & 1.0000 & 0.0000 \\
\hline & 0.25 & 7.4265 & 0.0923 & 1.0198 & 1.0299 \\
\hline & 0.50 & 6.8181 & 0.1941 & 1.0847 & 1.2921 \\
\hline & 0.75 & 4.5282 & 0.3174 & 1.2137 & 1.4557 \\
\hline & 1.00 & 2.1940 & 0.4830 & 1.4499 & 1.5431 \\
\hline & 1.25 & 0.6760 & 0.7383 & 1.9005 & 1.5473 \\
\hline & 1.50 & 0.0570 & 1.2343 & 2.9128 & 1.4463 \\
\hline \multirow{7}{*}{4} & 0.00 & 0.0000 & 0.0000 & 1.0000 & 0.0000 \\
\hline & 0.25 & 7.5641 & 0.0960 & 1.0210 & 1.0540 \\
\hline & 0.50 & 6.4826 & 0.2028 & 1.0905 & 1.2526 \\
\hline & 0.75 & 4.0844 & 0.3345 & 1.2308 & 1.3729 \\
\hline & 1.00 & 1.8775 & 0.5164 & 1.4941 & 1.4343 \\
\hline & 1.25 & 0.5418 & 0.8109 & 2.0185 & 1.4309 \\
\hline & 1.50 & 0.0396 & 1.4464 & 3.3162 & 1.3400 \\
\hline
\end{tabular}

\section{Results and Discussion}

In this section, the large deflection of the variable-arc-length beam made from nonlinear material subjected to the uniform self-weight is presented. According to the experimental data $[14,15]$, the stress-strain relation of some metals or hardening materials can be represented by the Ludwick constitutive law with the constant $n>1$. Therefore, the numerical examples are focused on the case of $n>1$ only.

Some numerical solutions of $\widehat{w}, \widehat{y}_{\max }, \widehat{s}_{t}$, and $\widehat{M}_{\max }$ for different values of $\theta_{A}$ with various values of the material constant $n$ (i.e., $n=1,2,3$, and 4 ) are presented in Table 1 . Figure 3 shows the load-deflection curves between the uniform self-weight $\widehat{w}$ and the end rotation $\theta_{A}$ for $n=1,1.5,2,3$, and 4 . As can be observed from Figure 3, the load-deflection curves are not monotonic but they have a turning point (or a critical point), where the stability can be exchanged at that point. The equilibrium path always emanates from the origin point, where the uniform self-weight $\widehat{w}$ equals zero. The uniform self-weight is developed as the deflection 


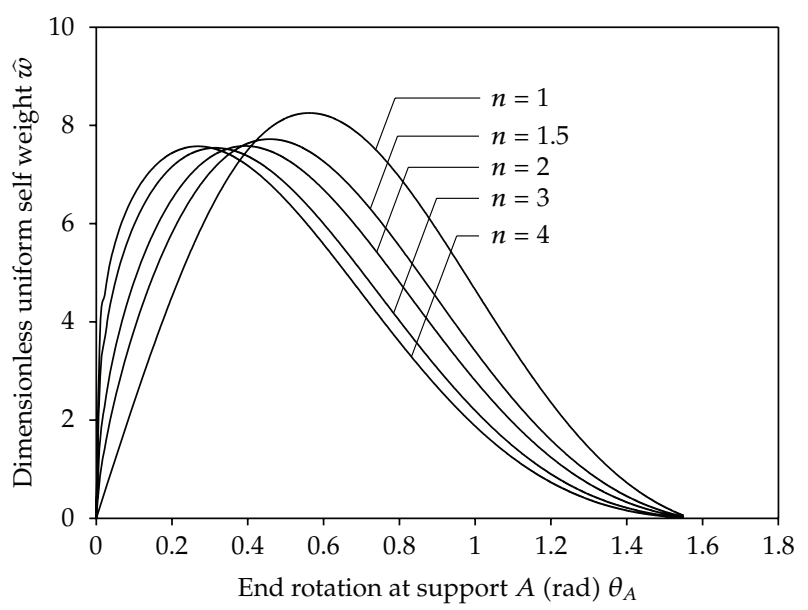

Figure 3: Variations of the dimensionless uniform self-weight $\widehat{w}$ with respect to the end rotation $\theta_{A}$ for $n=$ $1,1.5,2,3$, and 4 .

is increased, where the stable behavior can be captured (i.e., positive slope of load-deflection curves). The increase of the uniform self-weight is limited at the critical point, where the slope of load-deflection curves becomes zero. Afterwards, the deflection is increased while the uniform self-weight is decreased. This indicates the unstable behavior of the VAL beam (i.e., negative slope of load-deflection curves). Hence, we can state that, for a given value of $\widehat{w}$, there are two possible equilibrium configurations if the uniform self-weight is less than the critical value. Figure 4 illustrates two equilibrium configurations, which are the stable and unstable equilibrium configurations, for $\widehat{w}=5$ and $n=1,2,3$, and 4 . It is also found that the flexibility of the beam decreases, while the value of $n$ increases as shown in Figures 3 and 4 .

Figure 3 also shows that there is a critical value of $\widehat{w}$ corresponding with each value of $n$. These critical values are referred to the critical uniform self-weight $\widehat{w}_{\text {cr }}$ of the beam with a specific value of the material constant $n$. For the case of the linear elastic material $(n=1)$, the critical uniform self-weight of the variable-arc-length beam is equal to 8.2529 , which is identical to the value presented in [8]. Figure 5 illustrates the relationship between the critical uniform self-weight and the material constant $n$. It is found that the critical uniform selfweight $\widehat{w}_{\text {cr }}$ approaches to the minimum value of 7.5402 when the material constant $n$ is equal to 2.75 .

\section{Conclusions}

The large deflection of the variable-arc-length beam made from nonlinear material subjected to its uniform self-weight is presented. The nonlinearities of this problem can be arisen from both material and geometry of the VAL beam. The Ludwick constitutive law is utilized to represent the stress-strain relation of the beam material. The shooting method is employed to evaluate the numerical results. The numerical solutions indicate that there is a maximum value of $\widehat{w}$ for each value of $n$, which is referred to the critical uniform self-weight $\widehat{w}_{\text {cr }}$ of the beam with a specific value of the material constant $n$. For $\widehat{w}<\widehat{w}_{\text {cr }}$, there are two possible equilibrium configurations. The smaller displacement configuration is stable, while the larger one is unstable. The increase in value of $n$ reduces the flexibility of the beam. An interesting 


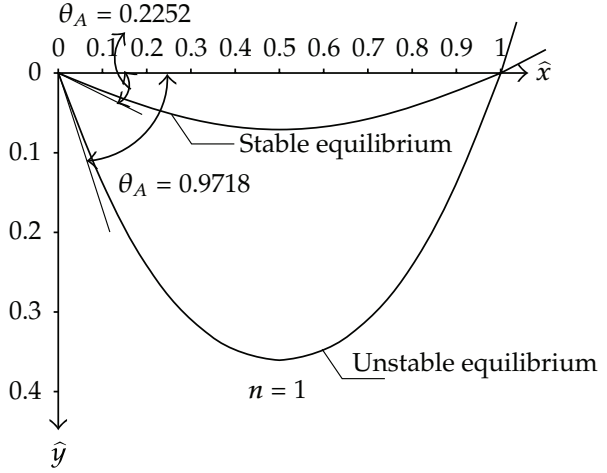

(a)

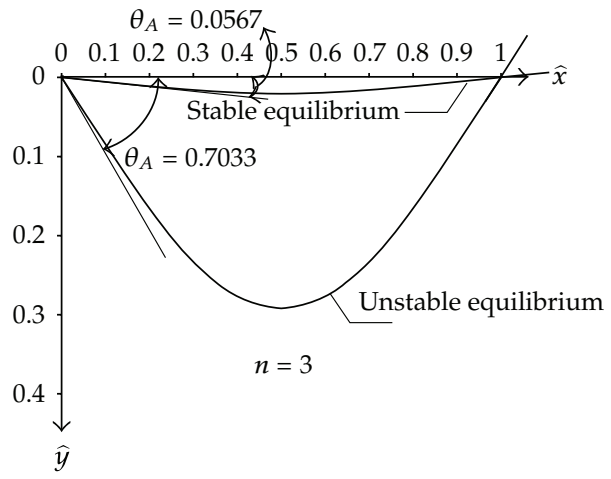

(c)

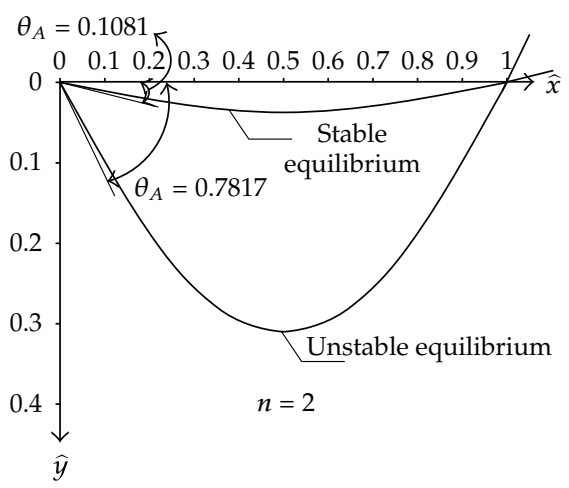

(b)

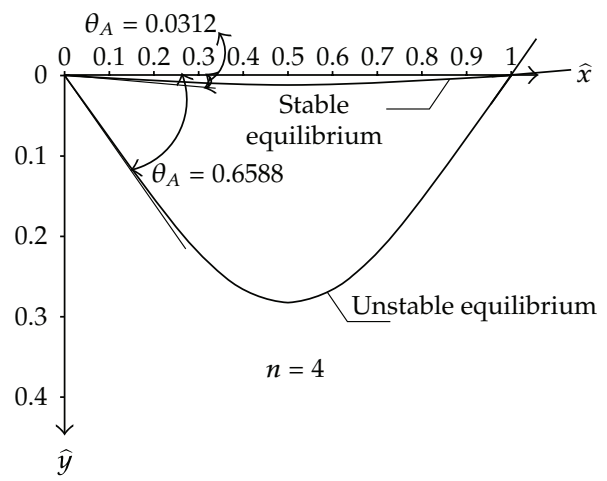

(d)

Figure 4: Equilibrium configurations of the variable-arc-length beams for $n=1,2,3$, and 4 subjected to the dimensionless uniform self-weight $\widehat{w}$ equal to 5 .

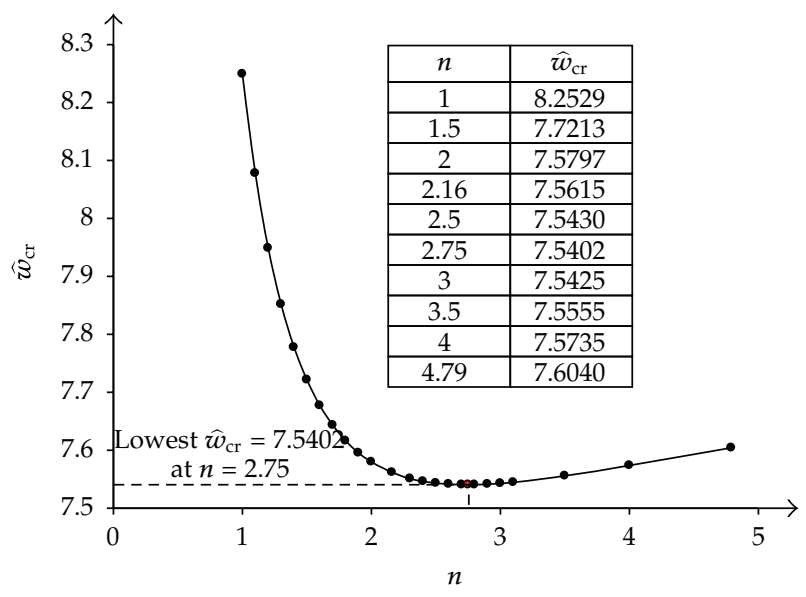

Figure 5: Variations of the critical dimensionless uniform self-weight $\widehat{w}_{\mathrm{cr}}$ with respect to the material property $n$. 
result also found in this study is that the critical uniform self-weight $\widehat{w}_{\text {cr }}$ approaches the minimum value when the material constant $n$ is equal to 2.75 , which is an important information for the practical engineers who work in offshore engineering and in the design of marine risers or cables.

\section{Acknowledgments}

The authors gratefully acknowledge the financial support by the Computational Science and Engineering Research Cluster (CSEC), King Mongkut's University of Technology Thonburi (KMUTT).

\section{References}

[1] S. Chucheepsakul, S. Buncharoen, and T. Huang, "Elastica of simple variable-arc-length beam subjected to end moment," Journal of Engineering Mechanics, vol. 121, no. 7, pp. 767-772, 1995.

[2] S. Chucheepsakul, G. Thepphitak, and C. M. Wang, "Large deflection of simple variable-arc-length beam subjected to a point load," Structural Engineering and Mechanics, vol. 4, no. 1, pp. 49-59, 1996.

[3] S. Chucheepsakul, G. Thepphitak, and C. M. Wang, "Exact solutions of variable-arc-length elasticas under moment gradient," Structural Engineering and Mechanics, vol. 5, no. 5, pp. 529-539, 1997.

[4] S. Chucheepsakul, C. M. Wang, X. Q. He, and T. Monprapussorn, "Double curvature bending of variable-arc-length elasticas," Journal of Applied Mechanics, vol. 66, no. 1, pp. 87-94, 1999.

[5] C. M. Wang, K. Y. Lam, X. Q. He, and S. Chucheepsakul, "Large deflections of an end supported beam subjected to a point load," International Journal of Non-Linear Mechanics, vol. 32, no. 1, pp. 63-72, 1997.

[6] T. Pulngern, M. W. Halling, and S. Chucheepsakul, "Large deflections of variable-arc-length beams due to uniformly distributed loading: analytical and experimental," Structural Engineering and Mechanics, vol. 19, pp. 413-423, 2005.

[7] X. Zhang and J. Yang, "Inverse problem of elastica of a variable-arc-length beam subjected to a concentrated load," Acta Mechanica Sinica, vol. 21, no. 5, pp. 444-450, 2005.

[8] C. Athisakul and S. Chucheepsakul, "Effect of inclination on bending of variable-arc-length beams subjected to uniform self-weight," Engineering Structures, vol. 30, no. 4, pp. 902-908, 2008.

[9] G. Lewis and F. Monasa, "Large deflections of cantilever beams of nonlinear materials," Computers and Structures, vol. 14, no. 5-6, pp. 357-360, 1981.

[10] G. Lewis and F. Monasa, "Large deflections of cantilever beams of non-linear materials of the Ludwick type subjected to an end moment," International Journal of Non-Linear Mechanics, vol. 17, no. 1, pp. 1-6, 1982.

[11] K. Lee, "Large deflections of cantilever beams of non-linear elastic material under a combined loading," International Journal of Non-Linear Mechanics, vol. 37, no. 3, pp. 439-443, 2002.

[12] M. Brojan, T. Videnic, and F. Kosel, "Large deflections of nonlinearly elastic non-prismatic cantilever beams made from materials obeying the generalized Ludwick constitutive law," Meccanica, vol. 44, no. 6, pp. 733-739, 2009.

[13] M. Brojan, A. Puksic, and F. Kosel, "Buckling and post-buckling of a nonlinearly elastic column," Journal of Applied Mathematics and Mechanics, vol. 87, no. 7, pp. 518-527, 2007.

[14] M. Sathyamoorthy, Nonlinear Analysis of Structures, CRC Press, Boca Raton, Fla, USA, 1998.

[15] E. Solano-Carrillo, "Semi-exact solutions for large deflections of cantilever beams of non-linear elastic behaviour," International Journal of Non-Linear Mechanics, vol. 44, no. 2, pp. 253-256, 2009. 


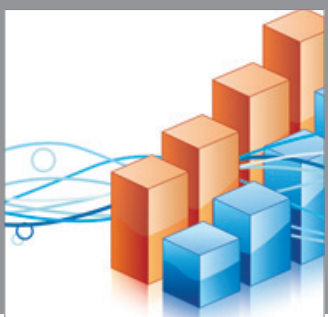

Advances in

Operations Research

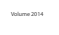

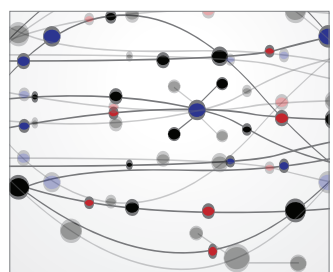

\section{The Scientific} World Journal
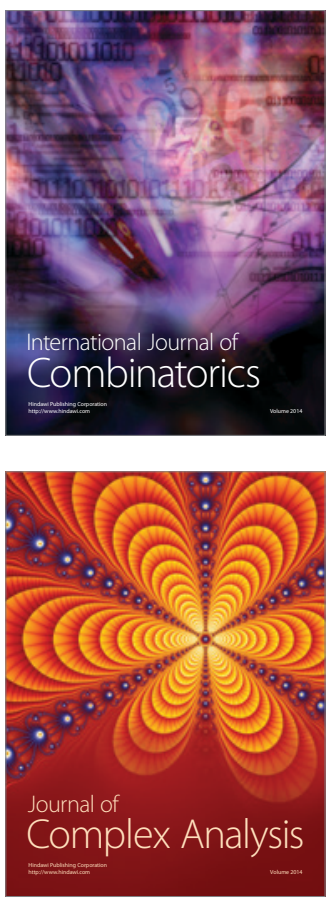

International Journal of

Mathematics and

Mathematical

Sciences
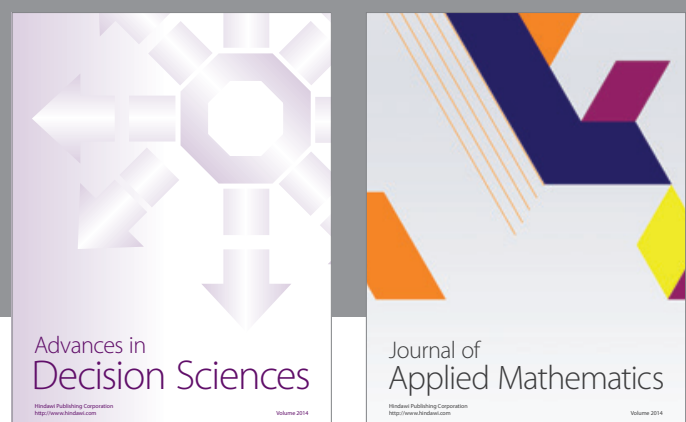

Journal of

Applied Mathematics
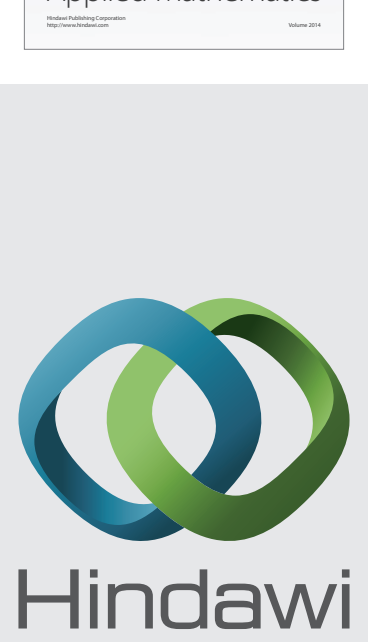

Submit your manuscripts at http://www.hindawi.com
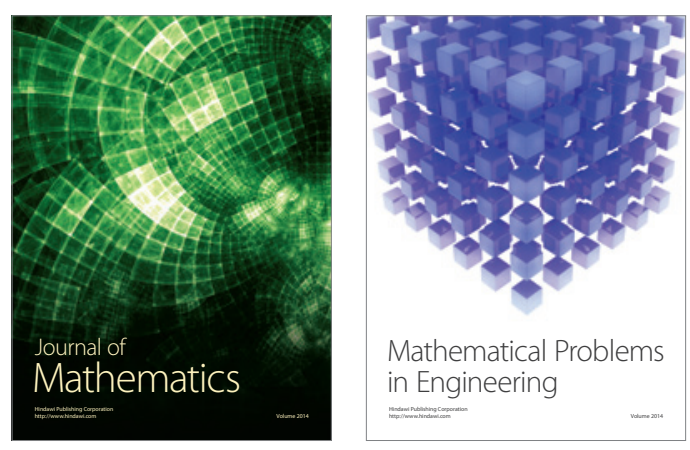

Mathematical Problems in Engineering
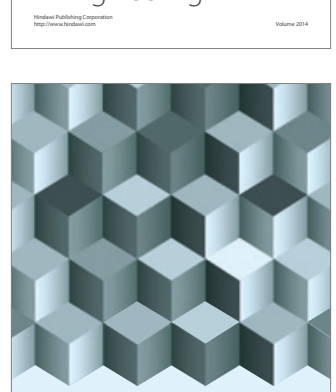

Journal of

Function Spaces
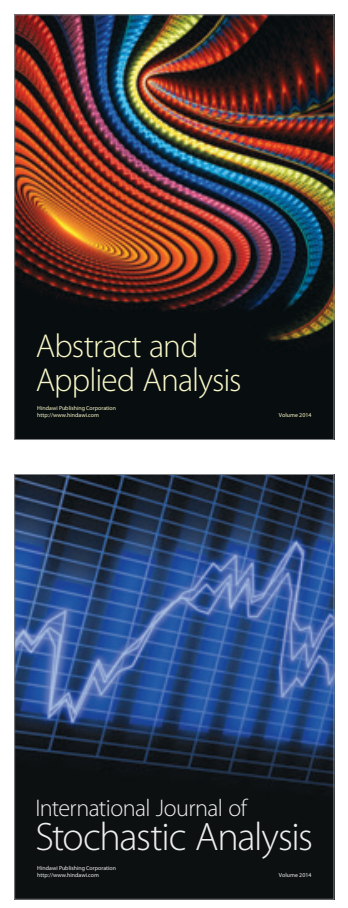

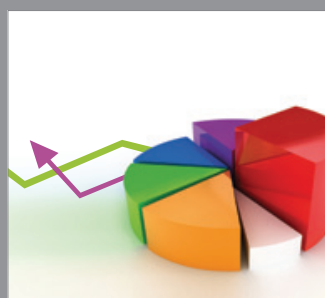

ournal of

Probability and Statistics

Promensencen
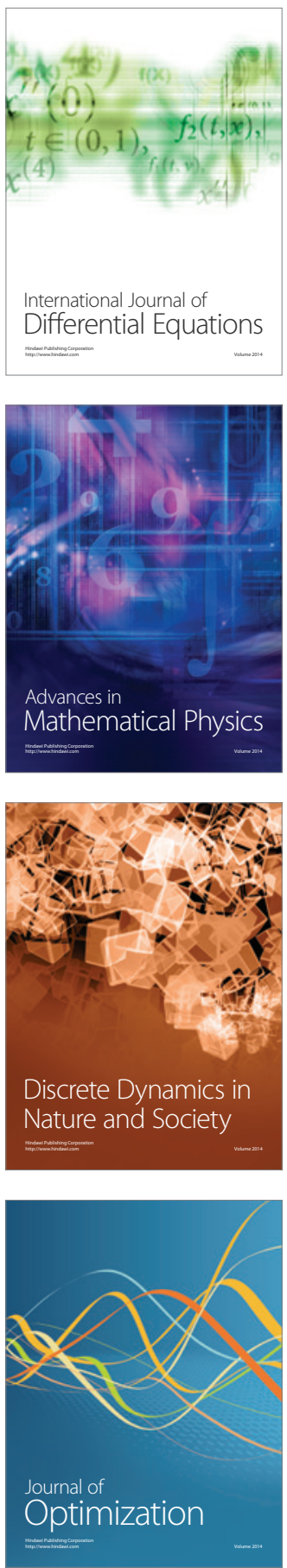